\title{
Adaptation of in vitro cytoadherence assay to Plasmodium knowlesi field isolates
}

\author{
Farrah A Fatih ${ }^{1 *}$, Angela Siner ${ }^{2}$, Atique Ahmed ${ }^{2}$, Lu Chan Woon ${ }^{3}$, Alister Craig ${ }^{4}$, Henry M Staines ${ }^{1}$, Balbir Singh ${ }^{2}$, \\ Sanjeev Krishna ${ }^{1,2}$, Janet Cox-Singh ${ }^{1,2}$ \\ From Parasite to Prevention: Advances in the understanding of malaria \\ Edinburgh, UK. 20-22 October 2010
}

\section{Background}

P. knowlesi was the first Plasmodium species in which antigenic variation was observed. Variation was due to schizont infected cell agglutination (SICAvar) antigens expressed by the parasite and transported to the exposed surface of the host erythrocyte [1]. PfEMP1 is $P$. falciparum's orthologue of $P$. knowlesi's SICA proteins [2]. In P. falciparum PfEMP1 is associated with infected erythrocytes binding to receptors such as ICAM-1 expressed on the endothelial cells of the host microvasculature. Here, we use a static protein assay [3] to determine if naturally occurring human $P$. knowlesi infections can cause erythrocytes to bind to ICAM-1, VCAM-1 and CD36.

\section{Materials and methods}

Blood samples were collected after obtaining written consent from patients presenting with $P$. knowlesi infection at two well-established study sites in Sarawak, Malaysian Borneo. The samples were washed and cultured ex vivo until the majority of parasites had matured to late trophozoite/early schizont stages of development. The parasites were then assayed for their ability to bind potential endothelial ligands. Static assays were carried out with purified proteins, ICAM-1, VCAM-1 and CD36. P. falciparum (cloneHB3) was used as a positive control.

\section{Results}

The relative binding of knowlesi-patient isolates compared with P. falciparum control assays will be presented.

\section{Conclusion}

The results of the binding assays will be presented and discussed in the context of recently described post-mortem findings from a fatal case of $P$. knowlesi.

\section{Acknowledgements}

The authors would like to acknowledge the contributions of staff at Hospitals Sarikei and Sibu, especially Mr Wong Ching Toh, Mr Pek Peng Chin, Madame Siti Syartinah and Mr Raymand Johan; and the staff at the Malaria Research Centre at UNIMAS. The authors also wish to thank the training provided by Mr Tadge Szestak at LSTM.

\section{Author details}

${ }^{1}$ Centre for infection, St George's University of London, London SW17 ORE, UK. ${ }^{2}$ Malaria Research Centre, University Malaysia Sarawak, Kuching, 93150, Malaysia. ${ }^{3}$ Pathology Laboratory, Hospital Sarikei, Sarikei, 96100, Malaysia. ${ }^{4}$ Molecular Biochemical Parasitology, Liverpool School of Tropical Medicine, L3 5QA, UK.

\section{Published: 20 October 2010}

\section{References}

1. Brown KN, Brown IN: Immunity to malaria: antigenic variation in chronic infections of Plasmodium knowlesi. Nature 1965, 208:1286-8.

2. Korir CC, Galinski MR: Proteomic studies of Plasmodium knowlesi SICA variant antigens demonstrate their relationship with P. falciparum EMP1. Infect Genet Evol 2006, 6:75-9.

3. McCormick CJ, Craig A, Roberts D, Newbold Cl, Berendt AR: Intercellular adhesion molecule-1 and CD36 synergize to mediate adherence of Plasmodium falciparum-infected erythrocytes to cultured human microvascular endothelial cells. J Clin Invest 1997, 100:2521-9.

doi:10.1186/1475-2875-9-S2-013

Cite this article as: Fatih et al:: Adaptation of in vitro cytoadherence assay to Plasmodium knowlesi field isolates. Malaria Journal 2010 9(Suppl 2):013. 